\title{
EFECTOS DE LA CORROSIÓN DEL ACERO DE REFUERZO EN LOS DIAGRAMAS MOMENTO-CURVATURA DE COLUMNAS DE CONCRETO RECTANGULARES
}

\author{
L. LANDA-RUIZ ${ }^{1,5}$, M. JARA-DÍAZ ${ }^{2}$, C. GAONA-TIBURCIO ${ }^{3}$, F. ALMERAYA-CALDERÓN ${ }^{3}$, \\ R. CROCHE ${ }^{4}$, S. MÁRQUEZ-MONTERO ${ }^{5}$, M. A. BALTAZAR-ZAMORA ${ }^{5}$ \\ ${ }^{1}$ Universidad Veracruzana, Doctorado en Ingeniería, FIME, Xalapa, Veracruz, México. \\ ${ }^{2}$ Universidad Michoacana de San Nicolás de Hidalgo, FIC, Morelia, Michoacán, México \\ ${ }^{3}$ Universidad Autónoma de Nuevo León. FIME - CIIIA. Av. Universidad S/N. \\ Ciudad Universitaria. San Nicolás de los Garza, Nuevo León, México \\ ${ }^{4}$ Universidad Veracruzana, FIME-Xalapa, Xalapa, Veracruz, México \\ ${ }^{5}$ Universidad Veracruzana, Facultad de Ingeniería Civil - Xalapa, Circ. G. Aguirre Beltrán S/N, \\ Lomas del Estadio, CP 91000, Xalapa, Veracruz, México
}

\section{RESUMEN}

En el presente trabajo se propone incluir en las curvas teóricas de momento-curvatura (m- $\square$ ) el efecto de la corrosión mediante la obtención de la disminución del área de acero de refuerzo longitudinal. La profundidad de corrosión se obtendrá a partir del ancho de grieta y longitud de corrosión observada en el recubrimiento de la columna. Con la profundidad de corrosion se obtendrá el área de acero que se pierde y esta modificación se incorporará al procedimiento teórico para elaborar las curvas m- $\square$. Los esfuerzos del acero se obtendrán de un modelo elastoplástico con endurecimiento curvo y los esfuerzos del concreto con un modelo que considere el efecto del confinamiento.

Palavras clave: Momento- Curvatura; Corrosión; Grieta; Resistencia de columnas.

\begin{abstract}
In the present work, we propose to include in the theoretical curves of moment-curvature the effect of corrosion by obtaining the reduction of the longitudinal reinforcement area. The corrosion depth will be obtained from the crack width and corrosion length observed in the cover concrete. With the depth of corrosion, the area of steel that is lost will be obtained and this modification will be incorporated into the theoretical procedure for making curves. The steel stress will be obtained from an elastoplastic model with curved hardening and the concrete stress with a model that considers the effect of confinement.
\end{abstract}

Keywords: Moment- Curvature relationship; Corrosion; Crack; Column Resistance.

\section{RESUMO}

No presente trabalho propõe-se incluir nas curvas teóricas de momento-curvatura o efeito da corrosão obtendo-se a redução da área de armadura longitudinal de aço. A profundidade de corrosão será obtida a partir da largura da fissura e do comprimento de corrosão observados no revestimento da coluna. Com a profundidade de corrosão, a área de aço perdida será obtida e essa modificação será incorporada ao procedimento teórico para a realização das curvas. Esforços de aço serão obtidos a partir de um modelo elastoplástico com endurecimento curvo e esforços concretos com um modelo que considera o efeito do confinamento.

Palavras-chave: curvatura do momento do relacionamento; Corrosão; rachar 


\section{INTRODUCCIÓN}

Los procedimientos de modelación de las articulaciones plásticas de los elementos de las estructuras de concreto reforzado consideran hipótesis del comportamiento de los materiales diferentes a las hipótesis utilizadas para el diseño del elemento, una de esas hipótesis se basa en modelar las curvas de esfuerzo - deformación de los materiales considerando los efectos del confinamiento y del deterioro del material, pero la reglamentación actual sólo considera el deterior del concreto por cargas cíclicas y con esas hipótesis se construyen los diagramas momento-curvatura que definen la articulación plástica. Si bien estas hipótesis se pueden usas en los casos donde se desea evaluar una estructura de concreto o realizar un análisis no lineal, éstas no reflejan el efecto del deterioro que el elemento de la estructura pueda tener por carbonatación del concreto, por perdida de la sección por agrietamiento o por corrosión del acero del acero de refuerzo, que es el principal causante del deterioro de las estructuras de concreto reforzado, cuyos mantenimientos por problemas de corrosión son de miles de millones de dólares tan sólo en E.U.A, muy en particular en puentes de concreto (Troconis et. al., 2016, Santiago et. al., 2016; Landa A.E et. al., 2018, Landa A.E et. al., 2018). Para mitigar el daño por corrosión se han realizado diversos trabajos de investigación, tomando en cuenta la calidad del concreto, tipo de cemento, aditivos, materiales alternativos al cemento portland, simulando medios agresivos de contacto como sulfatos y cloruros (Baltazar et. al., 2016; Santiago et. al., 2016; Landa A.E et. al., 2018, Landa A.E et. al., 2018). Por lo anterior se tiene la importancia del presente trabajo, en donde se evaluó el efecto que tiene la corrosión del acero de refuerzo sobre la gráfica esfuerzo - deformación del concreto confinado en columnas rectangulares. La construcción de la curva esfuerzo - deformación del concreto se hace siguiendo la propuesta de Mander et. al., (1988), la curva esfuerzo - deformación del acero con los valores propuestos por Mendoza et. al., (2013) y en una primera propuesta, considerando la pérdida de acero por corrosión de acuerdo con los criterios de Castonera (2007)

\section{RESISTENCIA DE LOS MATERIALES}

Normalmente las resistencias de los materiales, acero y concreto, que se consideran en un proyecto nuevo son conservadoras, para la modelación de las articulaciones plásticas mediante los diagramas momento - curvatura se deben adoptar valores que reflejen la resistencia real del material. Para ello en este trabajo se propone considerar los esfuerzos del acero con un modelo elastoplástico con endurecimiento curvo y para los esfuerzos del concreto con un modelo que considere el efecto del confinamiento.

\subsection{Resistencia del acero}

La curva esfuerzo deformación del acero será modelada con los parámetros propuestos por Medoza (2013) y que incorporan las Normas Técnicas Complementarias para Diseño y Construcción de Estructuras de Concreto (NTC, 2017) en la tabla 1 


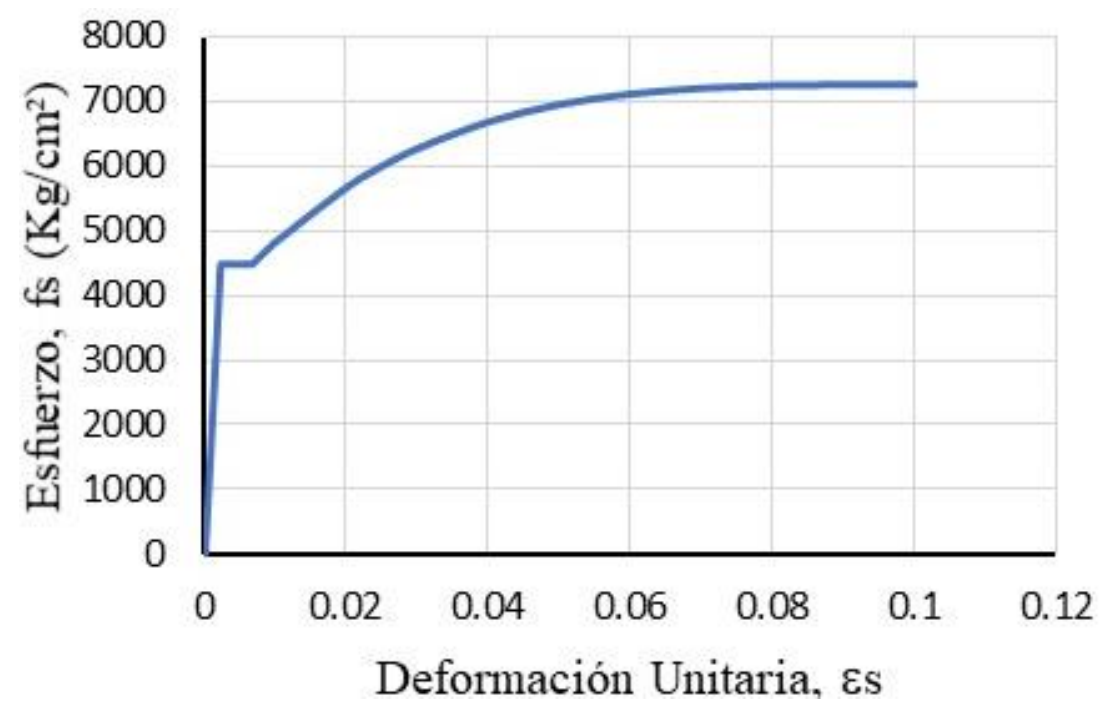

Figura 1. Curva esfuerzo - deformación del acero de refuerzo grado 42. Elaboración propia con los

\subsection{Resistencia del concreto}

criterios de las NTC (2017)

En la resistencia del concreto se tiene en cuenta el efecto del confinamiento proporcionado por el acero transversal, para lo cual la curva esfuerzo deformación se construye de acuerdo con la Figura 2 (Mander et. al, 1988) y que es un modelo teórico aceptado en las normas actuales (NTC, 2017)

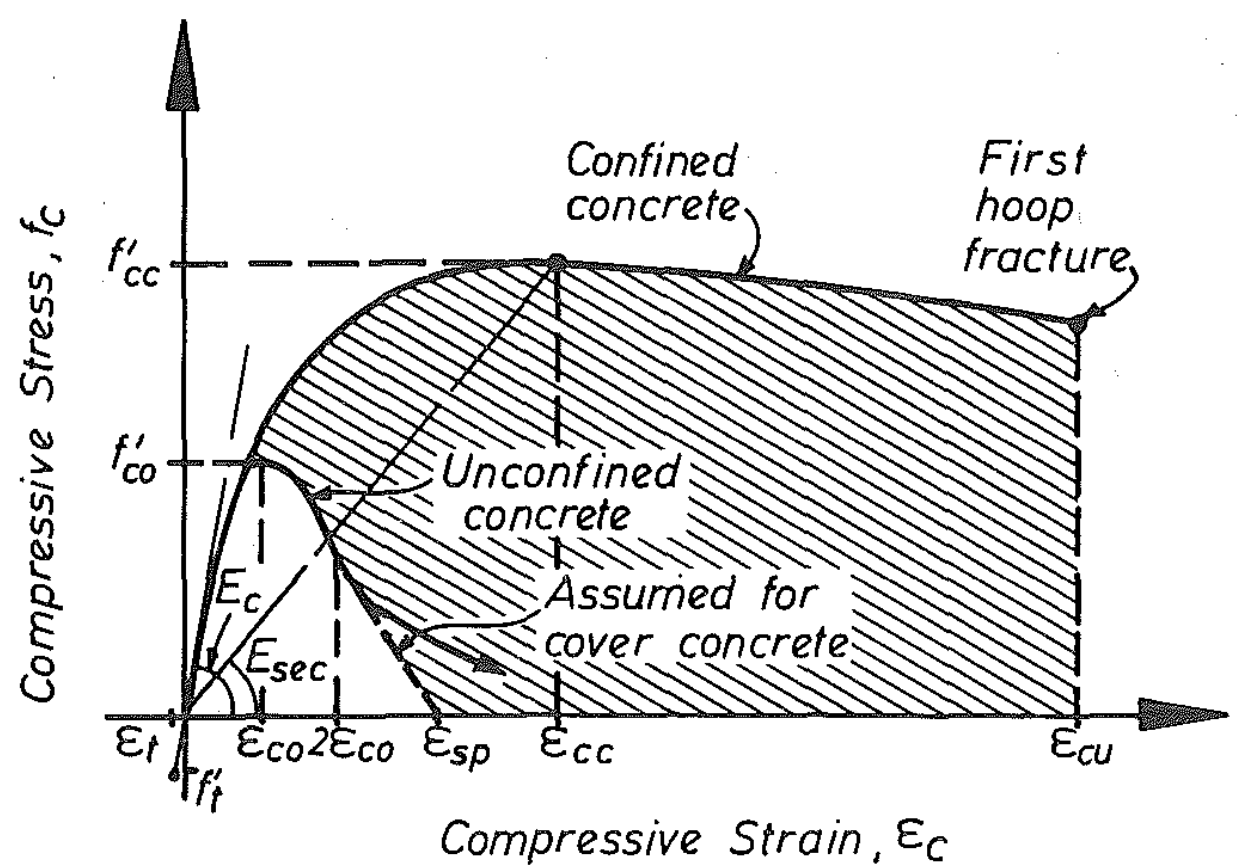

Figura 2. Curva esfuerzo - deformación del concreto (Mander et. al, 1988)

En la Figura 2, $f_{c o}^{\prime}$ es el esfuerzo de compresión del concreto simple, comúnmente identificado como $f_{c}^{\prime}, \varepsilon_{c o}$ es la deformación unitaria del concreto cuando se alcanza $f_{c o}^{\prime}$ que se considera de $0.003, f_{c c}^{\prime}$ el esfuerzo máximo de compresión considerando el confinamiento y $\varepsilon_{c c}$ es la deformación unitaria cuando alcanza dicho confinamiento. Para secciones rectangulares el esfuerzo máximo $f_{c c}^{\prime}$ se obtiene mediante la gráfica de la Figura 3 que involucra las relaciones de confinamiento $f_{l 1}^{\prime} / f_{c o}^{\prime}$ y $f_{l 2}^{\prime} / f_{c o}^{\prime}$ 


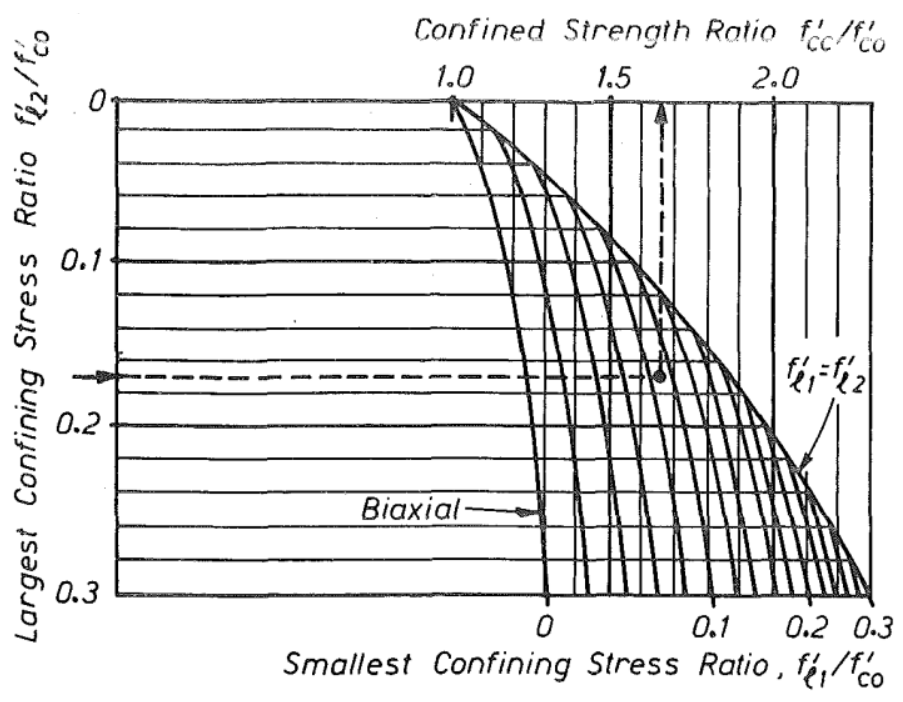

Figura 3. Determinación del esfuerzo de confinamiento a partir de las relaciones de confinamiento lateral para secciones rectangulares (Mander et. al, 1988)

$f_{l 1}^{\prime}$ y $f_{l 2}^{\prime}$ son el esfuerzo de confinamiento menor y mayor respectivamente en la dirección $\mathrm{X}$ o $\mathrm{Y}$ obtenidos con (1) que dependen de los porcentajes de acero longitudinal $\rho_{(x \text { ó } y)}$ y de un factor de confinamiento efectivo $k_{e}$.

$$
f_{l(x o ́ y)}^{\prime}=k_{e} \rho_{(x o ́ y)} f_{y h}
$$

$k_{e}$ a su vez, depende de la separación libre entre las barras longitudinales $w_{i}^{\prime}$, separación libre entre estribos $s^{\prime}$, distancia perpendicular a la dirección $\mathrm{x} b_{c}$, distancia perpendicular a la dirección y $d_{c} \mathrm{y}$ porcentaje de acero longitudinal $\rho_{c c}$. Ver figura 4 .

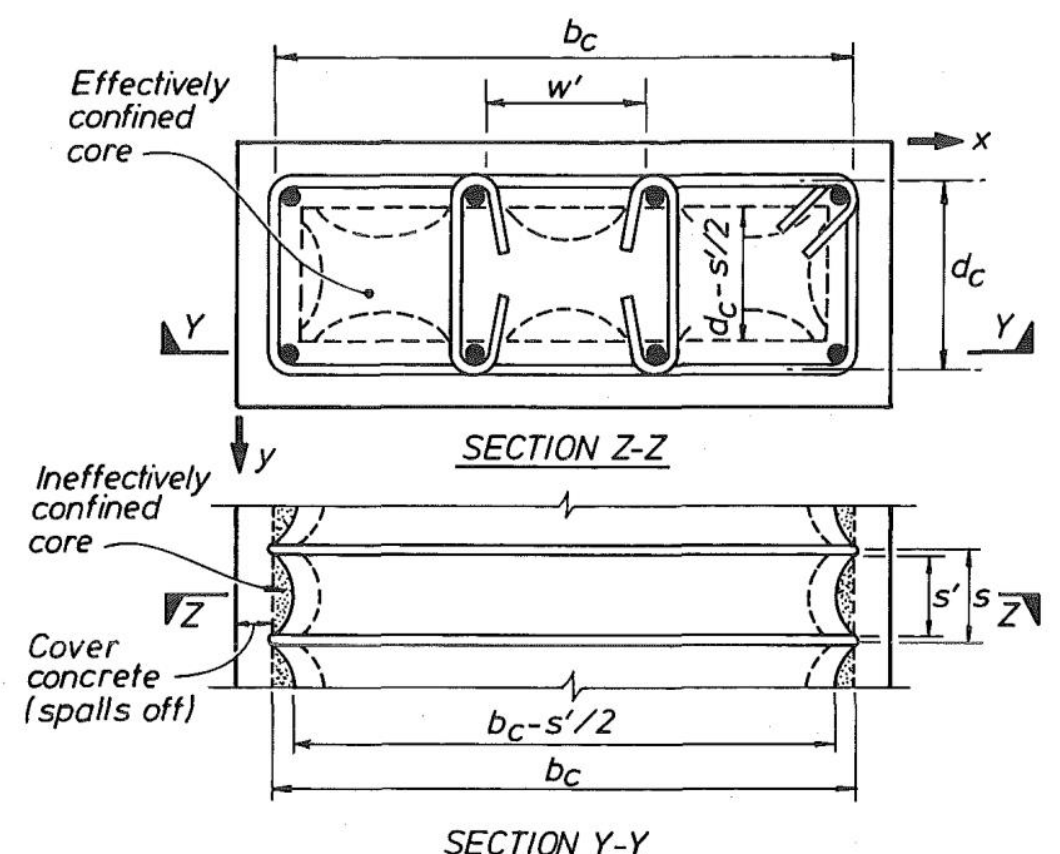

Figura 4. Núcleo confinado con refuerzo con estribos en una sección rectangular (Mander et. al, 1988) 
Para obtener el esfuerzo de confinamiento $f_{c c}^{\prime}$, de una manera menos visual que al usar la gráfica de la Figura 3 y nos permita auxiliarnos de una hoja de cálculo programable, se obtuvieron ecuaciones programables las cuales sustituyen la Figura 3 . Una vez obtenida las relaciones $f_{l 1}^{\prime} / f_{c o}^{\prime}$ y $f_{l 2}^{\prime} / f_{c o}^{\prime}$ se eligen dos de las ecuaciones obtenidas y con los dos resultados se interpola linealmente para finalmente obtener la relación $f_{c c}^{\prime} / f_{c o}^{\prime}$

\subsubsection{Deformación ultima del concreto}

Algunos autores (Priestley et. al., 1996) recomiendan que el valor que se adopte como deformación última del concreto $\varepsilon_{c u}$ sea de 0.005 para el caso del concreto pobremente confinado. Para el caso del concreto bien confinado, la deformación última puede obtenerse con (2) (Jara, 2004) derivada del criterio de balance de energía de Mander, también incluye la energía de deformación almacenada en el refuerzo longitudinal.

$$
\begin{aligned}
& \varepsilon_{c u}=\frac{110 \rho_{s}+3.4 \rho_{l}+0.017 \gamma \sqrt{f_{c}^{\prime}}}{\rho_{c c}\left(0.94 f_{y}+302\right)-\gamma\left(0.015 f_{c}^{\prime 2}-1.1 f_{c}^{\prime}-8\right)}\left(f_{c}^{\prime} y f_{y} \text { en } M P a\right) \\
& \gamma=0.45+0.5 \sqrt{\frac{P}{f_{c}^{\prime} A_{g}}}
\end{aligned}
$$

En (3) P es la fuerza axial y $A_{g}$ es la sección gruesa y la expresión sólo es aplicable para secciones rectangulares.
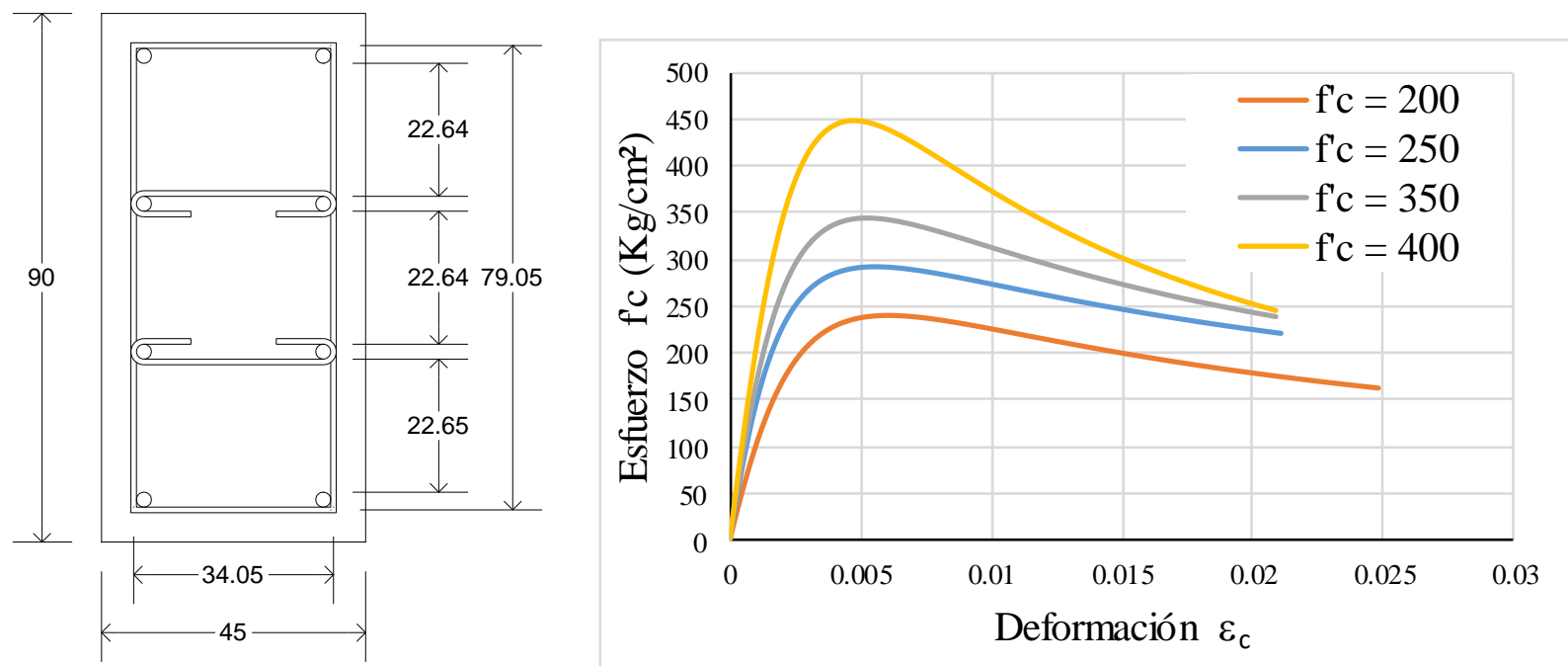

Figura 5. Diagrama esfuerzo-deformación para la columna mostrada a la izquierda. Elaboración propia

La Figura 5 muestra las curvas esfuerzo-deformación para diferentes $f_{c}^{\prime}$ de concreto siguiendo los criterios establecidos en el apartado 2 de este documento. Las curvas son específicas para la sección de la columna de la misma figura, que esta reforzada con 8 varillas del número 8 , estribos de varilla del número 3 con separación de $10 \mathrm{~cm}$.

\subsection{Obtención de los diagramas momento-curvatura (M- $\varphi)$}

Mediante un código propio desarrollado en el editor de Visual Basic para aplicaciones en Excel (VBA) se calcularon las curvas Momento - Curvatura siguiendo el procedimiento descrito en Nilson (1997) y Park y Pauly (1980), con las variaciones que la deformación ultima del concreto no será de 0.005 como 
lo proponen, si no la obtenida con (2). La fuerza de compresión del concreto $C_{c}$ se calcula con (4), donde b es la base de la sección de la columna y $f_{c c}^{\prime}$ se calcula con el criterio de Mander

$$
C_{c}=\propto f_{c c}^{\prime} b k d
$$

$\propto$ es un factor de esfuerzo medio (Park y Pauly, 1980) y se puede calcular con (5), donde el numerador representa el área bajo la curva esfuerzo-deformación del concreto, la cual en el código de VBA se realiza mediante incrementos de deformación unitaria y $\gamma$, es el factor del centroide para cualquier deformación $\varepsilon_{\mathrm{cm}}$

$$
\begin{aligned}
& \propto=\frac{\int_{0}^{\varepsilon_{c m}} f_{c} d_{\varepsilon_{c}}}{f_{c c}^{\prime} \varepsilon_{c m}} \\
& \gamma=1-\frac{\int_{0}^{\varepsilon_{c m}} \varepsilon_{c} f_{c} d_{\varepsilon_{c}}}{\varepsilon_{c m} \int_{0}^{\varepsilon_{c m}} f_{c} d_{\varepsilon_{c}}}
\end{aligned}
$$

Siguiendo el procedimiento descrito, se obtuvo el diagrama de la Figura 7, para un valor de f'c $=250$ $\mathrm{kg} / \mathrm{cm}^{2}$, la deformación última $\varepsilon_{c u}$ calculada con la fórmula (2) es de 0.02145

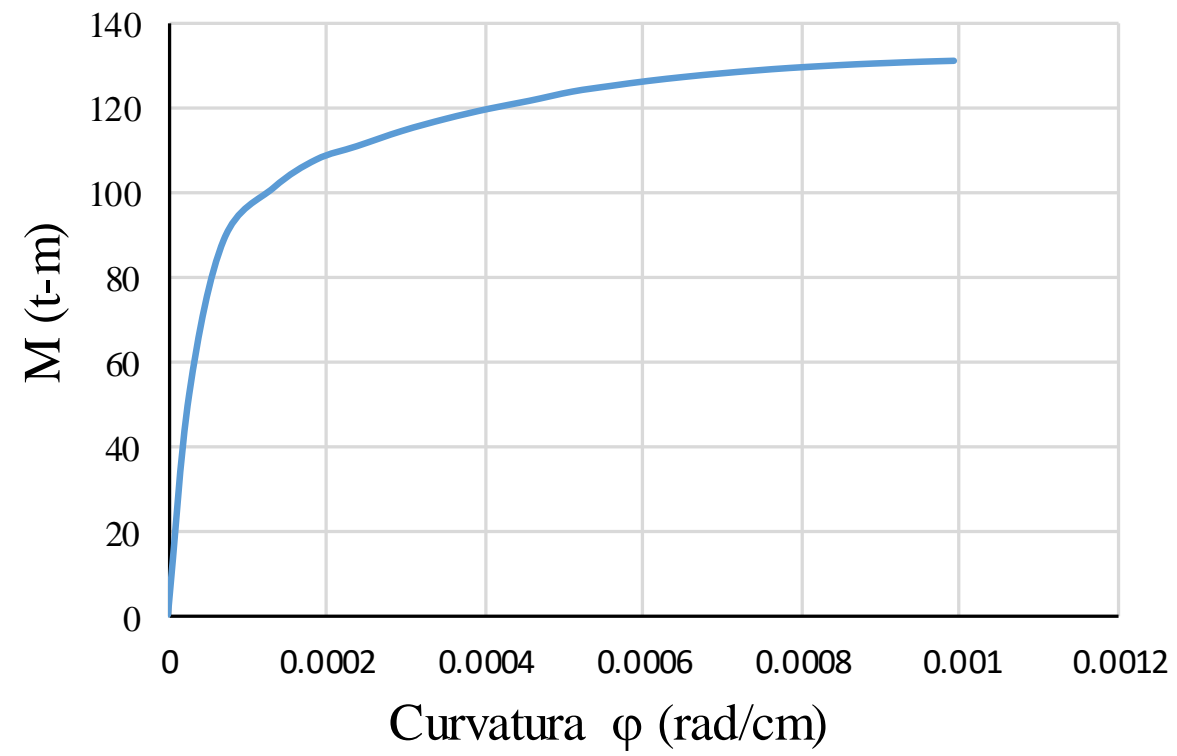

Figura 7. Diagrama momento curvatura específico para la sección de la Figura 5. Elaboración propia

\section{DAÑO POR CORROSIÓN}

Las curvas teóricas de momento - curvatura $(\mathrm{M}-\varphi$,$) son realizadas bajo las hipótesis ya establecidas$ anteriormente pero también bajo la suposición de que el acero de la columna está en condiciones óptimas, donde no presenta agrietamiento por corrosión o disminución del área de acero longitudinal debido a la corrosión. Cuando se desea evaluar una estructura de concreto las condiciones de conservación de la estructura pueden ser no las óptimas, el caso específico de las columnas de los puentes de concreto que se localizan en ciudades costeras o tramos carreteros cercanos al mar presenta daño por corrosión, por lo cual al hacer una evaluación en la que sea necesario contar con el diagrama M- $\varphi$, no estaría representando las condiciones reales del elemento de concreto. 
Castorena (2007) en su proyecto de investigación doctoral propone un modelo para estimar la profundidad de la corrosión de las varillas de acero a partir del ancho de grieta por corrosión, que puede ser medida mediante una inspección en campo. El modelo involucra sólo características del elemento, es decir la columna y las propiedades mecánicas del concreto.

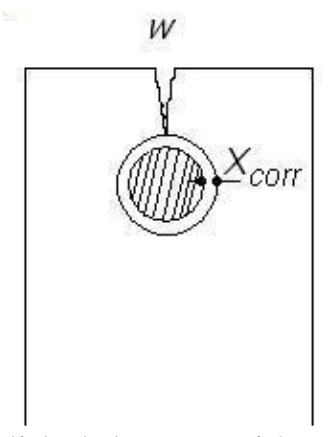

Figura 8. Profundidad de corrosión (Castorena, 2007)

El modelo propone que la profundidad de corrosión $X_{c o r r}$ está dada por la penetración sobre el acero al momento de iniciar el agrietamiento del recubrimiento por corrosión $X_{o}$ más la penetración en el acero después de que ha aparecido la primera grieta visible por corrosión $X_{p}$. Ver figura 8 .

$$
X_{\text {corr }}=X_{o}+X_{p}
$$

\subsection{Penetración de la corrosión al inicio del agrietamiento del recubrimiento por corrosión Xo}

Los resultados experimentales (Castorena, 2007) muestran que el ancho de la primera grieta por corrosión varía entre 0.06 y 0.08 milímetros, que es cuando se presenta la penetración por corrosión inicial $X_{o}$ y que podemos estimar con (8). $X_{o}$ depende de $C$ que es el valor del recubrimiento libre que tiene el acero, d el diámetro de la varilla y L longitud anódica, es decir, la longitud del acero corroído. Estas variables se muestran en la Figura 9

$$
X_{o}^{0.15}=0.4553\left(\frac{C}{d}\right)^{0.04} \delta^{-0.0653}\left(\frac{C}{L}\right)^{0.07}
$$

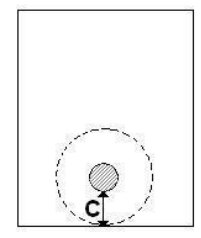

a)

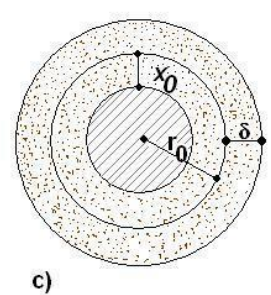

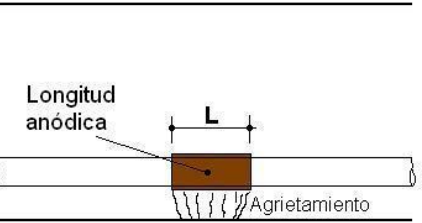

b)

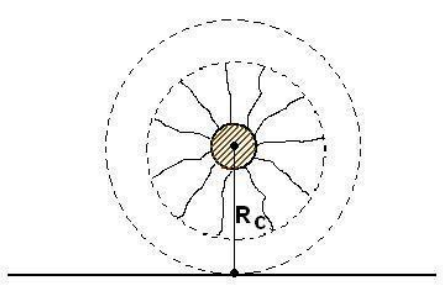

d)

Figura 9. Variables involucradas en la penetración de la corrosión al inicio del agrietamiento (Castorena, 2007) 
Se recomienda que la longitud anódica pueda identificarse plenamente utilizando alguna técnica electroquímica apropiada.

El parámetro $\delta$ está en función del radio de la varilla $r_{o}$, los módulos de poisson del concreto y del acero respectivamente $v_{c}$ y $v_{a}$, los módulos de elasticidad del acero y concreto $E_{a}$ y $E_{c}$ y de $R_{c}$ que es igual a la suma del radio de la varilla más el recubrimiento libre, como lo indica la Figura $9 \mathrm{~d}$ ). $\delta$ se obtiene con la fórmula (9) y representa la capa de óxido necesaria para producir esfuerzos tangenciales iguales a la resistencia máxima a tensión del concreto e iniciar el agrietamiento en la interface acero-concreto.

$$
\delta=2 r_{o} P_{g}\left[\frac{1-v_{a}}{E_{a}}+\frac{\left(1+v_{c}\right) R_{c}^{2}+\left(1-v_{c}\right) r_{o}^{2}}{E_{c}\left(R_{c}^{2}-r_{o}^{2}\right)}\right]
$$

$P_{g}$ es la presión radial en la interface acero-concreto y se obtiene con la con la ecuación (10), donde $f_{t}$ es el esfuerzo a tensión del concreto y para nuestro estudio es calculado como lo establece las NTC (2017) en función de la clase de concreto que se esté utilizando.

$$
P_{g}=\frac{f_{t}}{\left[\frac{R_{C}^{2}+r_{o}^{2}}{R_{C}^{2}-r_{o}^{2}}+v_{c}\right]}
$$

Si fijamos los valores de la longitud de corrosión a $150 \mathrm{~mm}$ y el recubrimiento libre de $50 \mathrm{~mm}$ podemos ver en la Figura 10 como influye el diámetro de la varilla y la resistencia del concreto sobre la penetración de corrosión inicial $\boldsymbol{X}_{\boldsymbol{o}}$. Las varillas de diámetro menor y los concretos de resistencia menor son los que presentan valores más altos de longitud de corrosión inicial y para concretos con valor de f'c mayores a $290 \mathrm{~kg} / \mathrm{cm}^{2}$ el esfuerzo del concreto deja de ser una variable que afecte el valor de $\boldsymbol{X}_{\boldsymbol{o}}$.

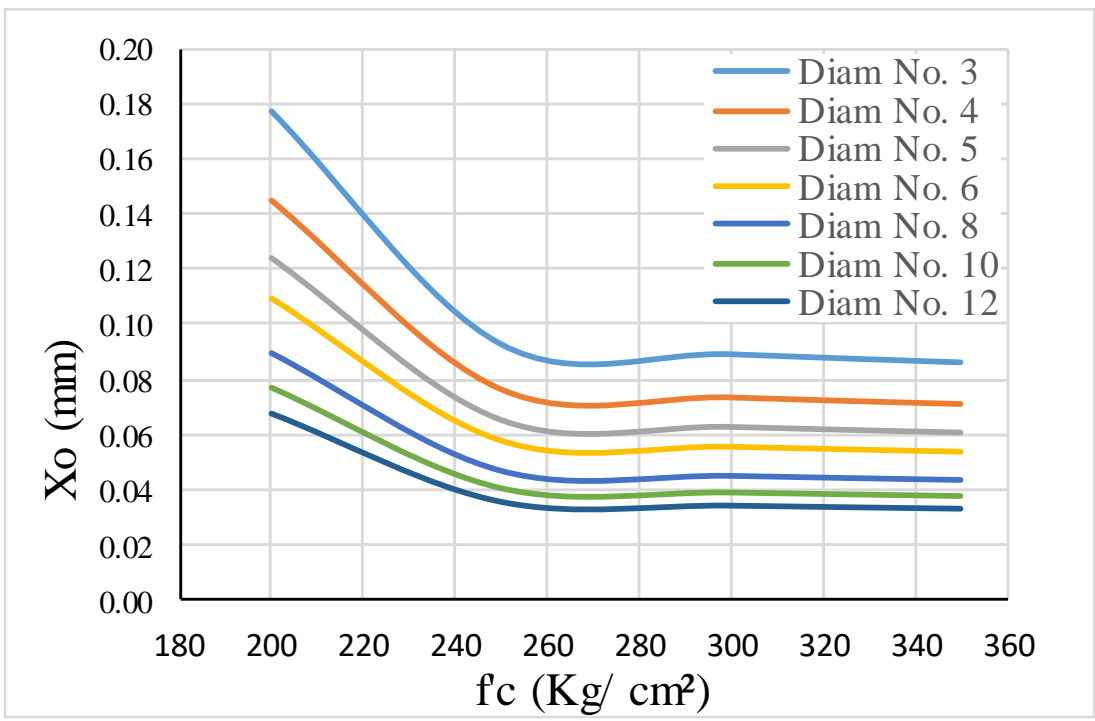

Figura 10. Penetración de la corrosión al inicio del agrietamiento del recubrimiento para diferentes valores de f'c y diferentes diámetros del acero. Elaboración propia

\subsection{Penetración de la corrosión después de que apareció la primera grieta visible por corrosión Xp}

Después de la aparición de la primera grieta por corrosión en el recubrimiento, la reducción del radio continúa dependido del ancho de grieta $w$ y se puede calcular con la fórmula (11) propuesta por Castorena (2007). En la figura 11 podemos ver la variación de $X_{p}$ para diferentes anchos de grieta. 


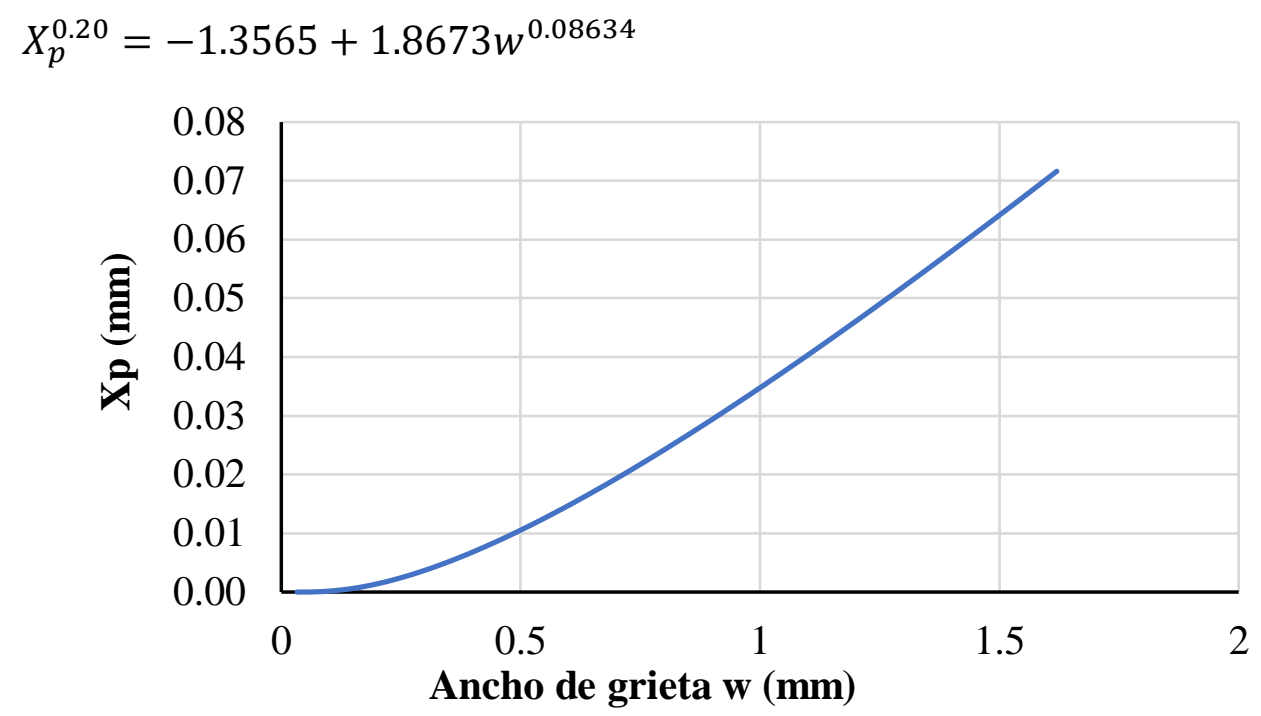

Figura 11. Penetración de la corrosión después de que apareciera la primera grieta por corrosión. Elaboración propia

En investigaciones realizadas (Vidal, et al., 2004) hay una notable diferencia en el ancho de grieta de los diferentes lados de la sección (sección frontal y sección posterior) de especímenes expuestos a ambientes marinos, esto debido principalmente a que la exposición a los agentes agresivos es diferente en los elementos estructurales dependiendo de su orientación, esto se puede observar en la Figura 12, los elementos de concreto presentan mayor daño por corrosión en una de las caras.

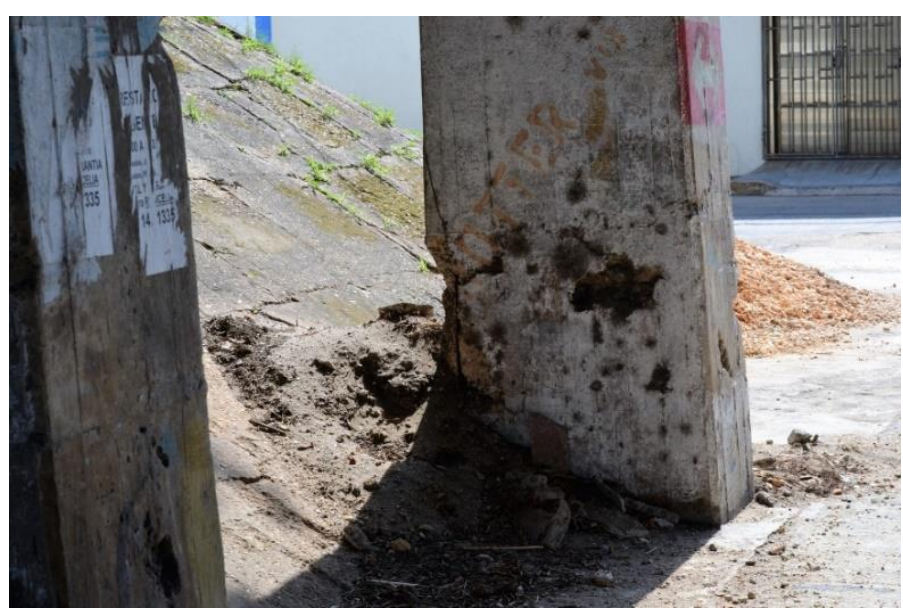

Figura 12. Columna del puente Palma Sola localizado en la Ciudad de Coatzacoalcos, Veracruz, México

\section{RESULTADOS}

Aplicando los criterios expuestos anteriormente, suponiendo una longitud de corrosión de $100 \mathrm{~mm}$, un $\mathrm{f}^{\prime} \mathrm{c}=250 \mathrm{~kg} / \mathrm{cm}^{2}$, diferentes anchos de grieta y una sección trasversal de las dimensiones de la Figura 5 , con varillas longitudinales del número 8 , estribos del número 3 a una separación de $10 \mathrm{~cm}$, obtenemos la Figura 13. 


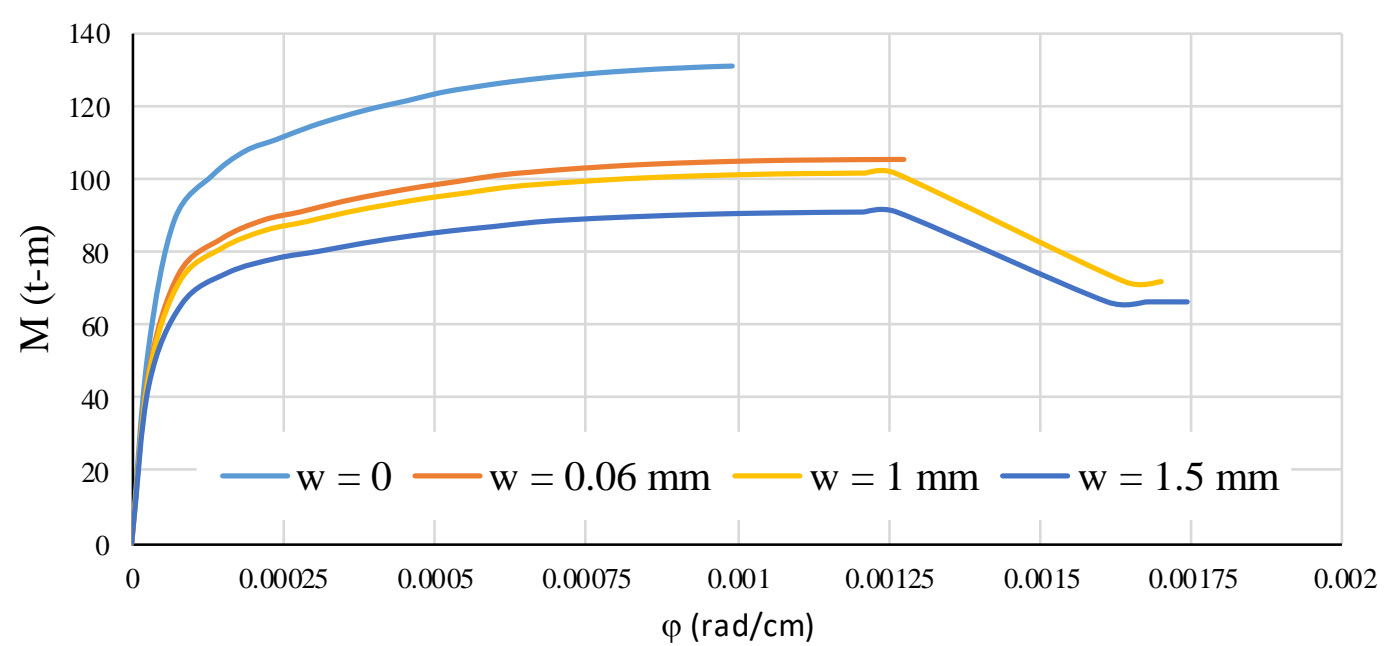

Figura 13. Diagrama momento curvatura de una sección rectangular bien con daños por corrosión estimados mediante el ancho de grieta. Elaboración propia

En la Figura 13, la curva para $\mathrm{w}=0$ corresponde a la sección de la Figura 5, considerando que el acero no ha sufrido daño por corrosión, las otras tres curvas mostradas en la Figura 5 son considerando que el acero longitudinal en sólo una de las caras ya presenta daño por corrosión y éste es estimado según el ancho de la grieta, en este caso se supusieron anchos de grieta de $0.06 \mathrm{~mm}, 1 \mathrm{~mm}$ y $1.5 \mathrm{~mm}$. Al aparecer la primera grieta por corrosión en el recubrimiento de un acho aproximado de $0.06 \mathrm{~mm}$ la profundidad de la corrosión es de una distancia de $0.05288 \mathrm{~mm}$ y corresponde sólo a $X_{o}$.

Tabla 1. Profundidad de corrosión para diferentes anchos de grietas de la sección de la Figura 5

\begin{tabular}{|c|c|c|c|c|}
\hline W (mm) & $\mathbf{X p}(\mathbf{m m})$ & $\mathbf{X o}(\mathbf{m m})$ & Xcorr $(\mathbf{m m})$ & \% de área de acero perdida \\
\hline 0.06 & 0.0000 & 0.0529 & 0.0529 & 65.97 \\
\hline 1 & 0.0348 & 0.0529 & 0.0877 & 90.40 \\
\hline 1.5 & 0.0641 & 0.0529 & 0.1170 & 99.38 \\
\hline
\end{tabular}

En la Figura 13 observamos como el diagrama M- $\varphi$, se ve afectado por el daño por corrosión que pueda presentar el elemento. La mayor disminución en el momento se da cuando aparece la primera grieta, reduciendo el momento máximo aproximadamente un 20\%. También podemos observar que la curvatura última se incrementa al aumentar el ancho de grieta a pesar de que el porcentaje de perdida de acero por varilla afectada es del orden de $90 \%$ (Ver tabla 1) para anchos de grieta mayores.

\section{CONCLUSIONES}

De los resultados obtenidos de la presente investigación se tienen las siguientes conclusiones:

- En un proceso de evaluación de estructuras de concreto mediante un análisis no lineal donde se requieran los diagramas de momento-curvatura para la modelación de la articulación plástica se debe considerar las aproximaciones teóricas que representen las condiciones reales del elemento, en cuanto a materiales y conservación de la estructura.

- Es viable el uso de la propuesta de Castorena para estimar la reducción de radio de acero por corrosión, ya que su propuesta requiere solamente de la observación en sitio del ancho de grieta, de datos elementales de la geometría e información y los materiales de la sección de concreto.

- Incluir el daño por corrosión de los diagramas momento-curvatura nos describe un mejor comportamiento del elemento, sobre todo para fines de evaluación, ya que el elemento 
teóricamente puede presentar una disminución del 20\% del momento al aparecer la primera grieta comparado con el que momento que alcanzaría si la sección no presentará corrosión.

\section{AGRADECIMIENTOS}

M.A. Baltazar-Zamora, et. al., agradecen a PRODEP por el Apoyo otorgado por la SEP, al Cuerpo Académico UV-CA-458 "Sustentabilidad y Durabilidad de Materiales para la Infraestructura Civil", en el marco de la Convocatoria 2018 para Fortalecimiento de Cuerpos Académicos con IDCA 28593.

\section{REFERENCIAS}

Baltazar-Zamora M.A., Santiago, G., Moreno V., Croche R., De la Garza M., Estupiñan, F., Zambrano, P., Gaona, C. (2016). Electrochemical Behaviour of Galvanized Steel Embedded in Concrete Exposed to Sand Contaminated with NaCl. Int. J. Electrochem. Sci. 11, 10306-10319, doi: 10.20964/2016.12.28. Castorena J. (2007), "Daño por corrosión en estructuras de concreto reforzado a partir del ancho de grieta", Tesis Doctoral, Centro de Investigación en Materiales Avanzados, p, 100.

Castorena, J., Almeraya, C., Velásquez, I., Gaona, C., Villafañe, A. (2005). "Modelación con elemento finito del agrietamiento por corrosión en vigas de concreto reforzado utilizando elementos de contacto mediante ANSYS". Conpat 2005, ed., VIII Congreso Latinoamericano de Patología de la Construcción y X Congreso de Control de Calidad en la Construcción, Asunción, Paraguay, 19-21 septiembre, Vol. I: Control de Calidad

Jara, M. (2004), "Procedimiento de diseño sísmico basado en desplazamientos, para puentes con aisladores de base histeréticos", Tesis Doctoral, Universidad Politécnica de Cataluña, Barcelona, España.

Landa A.E., Croche R., Márquez S., Galván R., Gaona C., Almeraya F., Baltazar-Zamora M.A. (2018). Correlation of Compression Resistance and Rupture Module of a Concrete of Ratio $w / c=0.50$ with the Corrosion Potential, Electrical Resistivity and Ultrasonic Pulse Speed, ECS transactions, 84, 217-227 Landa A.E., Croche R., Márquez S., Villegas R., Ariza H.A., Estupiñan F., Gaona C., Almeraya F., Baltazar-Zamora M.A. (2018). Corrosion Behavior 304 and 316 Stainless Steel as Reinforcement in Sustainable Concrete Based on Sugar Cane Bagasse Ash Exposed to $\mathrm{Na}_{2} \mathrm{SO}_{4}$, ECS transaction, 84, 179188

Landa A.E., Croche R., López O.M., Galván R., Cabral J.A., Gaona C., Almeraya F., Baltazar-Zamora M.A. (2018). Corrosion Behavior of AISI 316 Stainless Steel As Reinforcement in Ternary Sustainable Concrete Based on SCBA-SF Exposed in Seawater, ECS Meeting Abstracts, MA2018-02 584-584

Landa A.E., Castillo S., Reyes J.A., Villegas R., Galván R., Cabral J.A., Gaona C., Almeraya F., Baltazar-Zamora M.A. (2018). Evaluation of the Corrosion of AISI 304 Stainless Steel Embedded in Sustainable Concrete with High Volumes of SCBA-SF Exposed in Marine Environment, ECS Meeting abstracts, MA2018-02 587

Mander, J. B., Priestley, M. J. N. y Park, R. (1988), Teorical Stress-Strain model for confined concrete. Journals of Structural Engineering, ASCE, 114(8): 1804-1826

Mendoza, C. J., Aire, C., López, J., Hernández, F. (2013), Propiedades mecánicas de barras de refuerzo grados 4,52 y 56 . Instituto de Ingeniería, UNAM

Normas Técnicas Complementarias para el Diseño y Construcción de Estructuras de Concreto. (2017), Gaceta Oficial de la Ciudad de México.

Nilson, A. H. (1997). “Design of Concrete Structures", McGraw-Hill Companies

Park, R. y Paulay, T. (1980), "Estructuras de Concreto Reforzado”, Editorial Limusa, México.

Priestley, M. J. N., Seible, F., y Calvi, G. M. (1996), "Seismic Design and Retrofit of Bridges", A WileyInterscience Publication. Jonh Wiley \& Sons, Inc. 
Santiago, G., Baltazar-Zamora M.A., Galván, R., López, L.D., Zapata, F., Zambrano, P., A. Gaona, C. and Almeraya, F. (2016), Electrochemical evaluation of reinforcement concrete exposed to soil type sp contaminated with sulphates. Int. J. Electrochem. Sci. 11,4850-4864, doi: 10.20964/2016.06.31

Santiago, G., Baltazar-Zamora M.A., Olguín, J., López, L.D., Galván, R., Ríos, A. Gaona, C. and Almeraya, F. (2016), "Electrochemical evaluation of a stainless steel as reinforcement in sustainable concrete exposed to chlorides". Int. J. Electrochem. Sci. 11, 2994

Troconis de Rincon, O., et al. (2016), "Reinforced Concrete Durability in Marine Environments DURACON Project: Long-Term Exposure". Corrosion. 72, 824.

Vidal, T., Castel, A., Francois, R. (2004), "Analyzing crack width to predict corrosion in reinforced concrete". Cement and Concrete Composites. 22:407-415. 